der angegebenen Weise gab ein negatives Resultat. Die Verseifungszahlen des acetylirten und des ursprünglichen Wachses wurden genau gleich, nämlich zu 222 gefunden.

Bell erbielt bei der Verseifung des Kuhbutterfettes mit nnzureichender alkoholiseher Kalilauge ein bei $4,4^{\circ}$ schmelzendes ÖI, welches er für ein Diglycerid hielt. Wir haben den Versuch wiederholt und in der That ein dünnflüssiges, stark nach Buttersäureäther riechendes ÖI exhalten, welches jedoch bei der Verseifung keine nachweisbare Glycerinmenge lieferte und zum grössten Theile aus Fettsäureäthylesterı bestand.

\section{Über die bei der Verwendung des Wassergases zu industriellen Zwecken erforderlichen Vorsichtsmassregeln.}

Ton

\section{G. Lunge.}

Durch den Fabrikinspector Dr. Schuler war das schweizerische Industrie-Departement auf die mit der Benutzung des Wassergases zu industriellen Zwecken verknüpften Möglichkeiten einer gesundheitlichen Schädigung der Arbeiter hingewiesen worden. Dies veranlasste das Departement zur Einsetzung einer Expertencommission, bestehend aus dem Verfasser (als Vorsitzenden), dem Dr. O. Wyss, Professor der Hygiene in Zürich und dem Director des Berner Gaswerkes, Herrn Rothenbach, mit Zuziehung der Fabrikinspectoren. Im Folgenden gebe ich den von mir verfassten Bericht der Commission an das Departement (mit Weglassung der formellen Einleitung) ferner das Gutachten des Prof. Wyss wieder, und bemerke schliesslich, dass die von uns vorgeschlagenen Massregeln auf Antrag des Industriedepartements von dem schweizerischen Bundesrathe genehmigt und in Form einer Verfügung in Bundesblatte veröffentlicht worden sind.

„Es muss in erster Linie festgestellt werden, dass unter dem Worte., Wassergas" von den Industriellen zwei ganz verschiedene Gasarten verstanden werden, wodurch einige Verwirrung angerichtet wird. Das wirkliche Wassergas wird erzeugt, indem man überhitzten Wasserdampf ohne Luftbeimengung durch glühenden Koks od. dgl. durchleitet, wobei ein Gemenge von ungefähr 50 Raumtheilen Wasserstoff, 40 Kohlenoxyd und 10 Kohlensäure, Stickstoff u. dgl, entsteht. Dieses Gas wird zur Erzeugung ganz hoher
Hitzegrade, sowie, mit Hinzuziehung anderer Factoren, zur Beleuchtung angewendet. Es ist in Amerika sehr verbreitet, aber in Luropa erst seit wenigen Jahren, in eigenartiger Ausbildung, eingeführt worden. In der Schweiz besteht bis jetzt erst ein einziger hleiner Apparat für lirzeugung an wirklichem Wassergas, nämlich bei Gebrüder Sulzer in Winterthur, welche denselben eigentlich nur als Versuchsapparat betrachten, und noch immer an demselben weiter studiren. is sind zwar von einer Seite her Versuche gemacht worden, das grössere Publicum dafür zu interessireu, dass das Wassergas zur öffentlichen Beleuchtung angeführt werde, aber einen Erfolg hat dies bisher nicht gehabt. Ehe an eine offentliche Benutzung des Wassergases zu Leucht- und Heizzwecken, analog dem gewöhulichen Leuchtgase, gedacht werden kann, müsste die Vorfrage gelöst sein, diesem an sich geruchlosen Gase auf irgend welchem Wege einen noch viel stärkeren Geruch als dem gewöhnlichen Leuchtgase zu ertheilen, da das Wassergas seines hohen Kohlenoxydgehaltes wegen mindestens fünfmal so giftig als das Leuchtgas ist, und seine Benutzung im geruchlosen $\mathrm{Zu}$ stande für das allgemeine Publicum dadurch ganz ausgeschlossen erscheint. Es wird zwar behauptet, dass man in allerneuester Zeit es dahin gebracht habe, dem Wassergase in der That einen penetranten und bleibenden Geruch zu ertheilen, aber wir brauchen dieser Frage erst später näher zu treten, da das wirkliche Wassergas zur Zeit in der Schweiz noch keine industrielle Verwendung gefunden hat, und im ferneren, weil die von uns für das sofort zu besprechende "Halbwassergas" empfohlenen Vorsichtsmassregeln auch auf das wirkliche Wassergas angewendet werden können und sollten.

Was in der Schweiz unter dem Namen "Wassergas" angewendet wird, ist, abgesehen von dem Sulzer'schen Falle, etwas ganz anderes, nämlich eiu Gas, velches dadurch erhalten wird, dass man in einen gewöhnlichen, mit natürlicher Zugluft oder Gebläse betriebenen "Gasgenerator" Wasser oder Wasserdampf zutreten lässt. Der Wasserdampf wird von der glühenden Kohle im Generator zersetzt, und mischen sich die Zersetzungsproducte dem gewöhnlichen Generatorgase bei. Man newnt daher dieses Gasgemenge in der Industrie "Mischgas" oder "Generatorwassergas" oder auch „Halbwassergas", welchen letzteren Ausdruck wir als den bezeichnendsten hier beibehalten wollen. Es gibt eine Anzahl von Systemen zur Erzeugung solchen Gases, welche sich jedoch 
nicht im Princip, sondern nur in den Einzelheiten unterscheiden. Dieselben haben in allen Industrieländern schon eine ausserordentlich grosse Verbreitung gefunden, und gelten als eines der rationellsten Feuerungssysteme für gewerbliche Zwecke. Tines dieser Systeme, dasjeuige von $\mathrm{D}$ ows on, ist auch in der Schweiz, aber bisher nur an sehr wenigen Orten eingefülnt worden. Man benutzt dasselbe in einer Hutfabrik zu Bendlikon zum Erhitzen der eisernen Hutformen, in einigen Floretspinnereien zum Sengen der Seide; an mehreren Orten zum Betrieb von Gasmotoren, und nebenbei zum Löthen, Kochen u. dgl. mehr.

Das Dowson-Gas, sowie die analogen Halbwassergase, hat vor dem Leuchtgas den Vorzug der grossen Billigkeit, und vor dem gewöhnlichen Generatorgase deujenigen einer grössereu Heizkraft; auch brennt es leichter als die letzten, dabei aber, ungleich dem Leuchtgase, mit nicht russender Flamme. Es eignet sich seiner Billigkeit wegen vorzüglich zum Betriebe von Gasmotoren, wobei man dann auch nicht von dem Vorhandeusein einer öffentlichen Gasanstalt abhängig ist. Seine Benutzung zum Sengen ist namentlich wegen der Eigenschaft des Nicht-Russens vortheilhaft. Nach den anderweitig gemachten Erfahrungen steht zu erwarten, dass auch in der Schweiz solche Halbwassergase in immer ausgedehntere Anwendung kommen werden. Dieses Gas wird übrigens in Röhrennetzen mit Hähnen u. dgl. genau nach Art der gewöhnlichen LeuchtgasLeitungen verwendet, und ist deshalb ein Entweichen desselben durch Undichtheit der Leitungen, Offenstehenlassen von Hähnen u. dgl. ebenso wie im letzteren Falle möglich. Das Halbwassergas besitzt, wenn es aus schwefelhaltigem Anthracit oder Koks gemacht ist, einen entschiedenen Geruch, der aber immerhin ungemein viel schwächer und weniger penetrant als derjenige von Leuchtgas ist, und bei Anwendung von schwefelarmem Material ist dieser Geruch sogar sehr unbedeutend.

Während nun in den übrigen Fabriken, wo das Dows on-Gas angewendet wird, keine Klagen laut geworden sind, hat Herr Dr. Schuler aus der Hutfabrik in Bendlikon häufige Klagen über Gesundheitsstörungen der Arbeiter erhalten, welche sich in Kopfweh, Schwindel, Übelkeit und Appetitlosigkeit äusserten. Nun muss bemerkt werden, dass freilich in jener Fabrik die Art der Anwendung des Gases eine solche war, dass sehr leicht eine erhebliche Menge desselben entweichen konnte. Das Gas strömte nämlich aus vielen sebr kleinen Öffnungen aus, wobei häufig einzelne der Flämmchen erloschen und unverbranntes Gas in die Luft gehen musste. Da die betreffenden Apparate gan\% frei in dem Saale stehen, ohue Abzug der Verbrennungsproducte, so mussten sich nicht nur diese, sondern auch das unverbraunte Gas der von den Arbeitern eingeathmeten Luft beimengen. Es war schon ein erheblicher Fortschritt, als auf Schuler's Veranlassuug die kleinen Löcher der Apparate durch lange Schlitze ersetzt wurden, deren Flammen nicht mehr ausgehen; aber auch dies bietet keinen absoluten Schutz gegen das Entweichen von unvollständig verbranntem Gas in die Luft des Fabriksaales.

Die aus der erwähnten Quelle stammenden Übelstände würden zwar fortfallen, wenn die Vorschrift erlassen würde, dass in allen Wassergas oder Halbwassergas benutzenden Fabriken die Verbrenuungsproducte, einschliesslich des möglicherweise unverbrannt aus den Brennern ausströmenden Gases, in solcher Weise abgeführt werden müssen, dass sie sich der zum Athmen dienenden Luft nicht beimischen können. Dies wirl dadurch erleichtert, dass das geringe specifische Gewicht des rohen Gases und die Hitze der Verbrennungsproducte beide dahin zielen, diese schädlichen Producte in die Höhe zu führen; wo also nicht die Brenner schon über Kopfhöhe angehracht sind, so kann man durch Trichter, die in's Freie oder in einen Schornstein führen, leicht für Abzug sorgen.

Aber die Commission glaubte ihre Aufgabe nicht mit der Empfehlung dieser Massregel erledigen zu dürfen, sondern hielt dafür, dass man die Frage untersuchen müsse, ob die Anwendung jener Gase überhaupt gesundheitliche Schädigungen hervorrufen und eventuell directe Tergiftungen dadurch veranlassen könne, dass die Gase leicht einmal durch Undichtheit von Rohrleitungen, Offenlassen von Hähnen u. dgl. frei in die Fabriklocale, auch vom Boden her, eindringen können.

Nun ist zwar allbekaunt, dass ganz ähnliche Gefahren auch bei dem, anstandslos ja nicht nur in Fabriken, sondern auch in Wohnungen seit Grenerationen verwendeten Leuchtgase bestehen. Aber es lässt sich nicht leugnen, dass bei den fraglichen Gasarten diese Gefahren aus doppeltem Grunde viel grösser sind: einmal weil diese Gase wenig oder unter Umständen gar keinen erheblichen Geruch haben, und daher eine Beimischung derselben zur Zimmerluft selbst in einem lebensgefährlichen Grade sich nicht, wie beim Leuchtgas, schon von selbst verräth; zweitens, weil diese Gase das Leucht- 
gas an Giftigkeit bei weitem übertreffen, wenigstens wenn man, nach Massgabe aller früheren Untersuchungen, die giftigen Wirkungen aller solcher Gase wesentlich von ihrem Gehalt an Kohlenoxyd herleiten will. Das gewöhnliche Leuchtgas enthält nämlich im Durchschnitt etwa 8 Proc., dagegen das wirkliche Wassergas etwa 40 Proc. und das Halbwassergas 22 bis 25 Proc. Kohlenoxyd. Ausserdem enthalten letztere in rohen (nicht besonders gereinigten) Zustande noch ein wenig Schwefelwasserstoff, doch ist dessen Menge so unbedeutend, dass seine toxischen Wirkungen neben denjenigen des Kohlenoxyds gar nicht in Betracht kommen, und auch in der That bei den Versuchen des Herrn Prof. Wyss gar nicht differenziirt werden konnten.

Die Literatur enthält eine grössere Anzall von Arbeiten über die gesundheitsschädlichen Wirkungen des Leuchtgases; aber es fehlen noch directe Versuche mit Wassergas und Halbwassergas. Die Commission glaubte daher, zur gründlichen Erledigung ihrer Aufgabe diese Lücke ausfüllen zu müssen, um keinen Zweifel über den Sachverhalt bestehen zu lassen, und übernalm Prof. Wyss die Ausführung der nötbigen Beobachtungen, insbesondere zahlreicher Thierversuche, in dem ihm unterstehenden Hygiene-Institut der Universität Zürich, während die Analysen der Gase von Prof. Lunge ausgeführt wurden. Über die erhaltenen Resultate hat Herr Prof. Wyss einen besorideren ausführlichen Bericht erstattet, den wir, sowie die Analysen der rerwendeten Gase, im Original beilegen. Die Schlussfolgerungen desselben sind folgende:

Das Halbwassergas sowie das Wassergas verdanken ilure Giftigkeit ihrem Gehalte an Kohlenoxyd. Eine Luft, welche 10 Prom. Wassergas oder 15 Prom. Dowsongas enthält, ist für Warmbliter tödtlich und Krankheitserscheinungen treten schon bei 1 Prom. Wassergas oder 3 Prom. Dowsongas ein. Die aus einem offenen Gashahn (von der für Leuchtbrenner gewöhnlichen Grösse) in eiver Stunde ausströmende Menge von Wassergas, genügt lei einem Zimmer von beiläufig $43 \mathrm{clbm}$ Inhalt, um die Insassen desselben comatös zu machen oder selbst zu tödten. Dabei wird schon frühzeitig das Bewusstsein gestört und dann aufgehoben. und werden die schützenden Reflexe vernichtet, so dass der Mensch bei der Geruchlosigkeit jener Gase, seiner natürlichen Hilfsmittel zur Walhrnehmung und Abwehrung der Gefahr beraubt wird.

Auf Grund vorstehender Resultate dürfen wir nunmehr in der That den Schluss aussprechen, dass dem wirklichen Wassergase etwa die fünffache, dem Dowson-Gas oder anderen Halbwassergasen etwa die dreifache
Giftigkeit des Leuchtgases zukommt, und dass beiden Gasen gerade wegen ihres unbedeutenden Geruches eine um so grössere Gefährlichkeit beiwolnt. Letzteres ist auch ein höchst wichtiger Unterschied gegenüber dem früher vielfach, jetzt fast gar nicht mehr verwendeten Holzgase, welches zwar ebenfalls ausserordentlich reich an Kohlenoxyd war, aber zugleich einen höchst penetranteu Geruch besass. Ebenso verhält es sich mit der Anwendung des Wassergases in Amerika, wo es in etwa 100 Gasanstalten zur öfentlichen Beleuchtung benutzt wird; dewn dort wird ihm die Leuchtkraft durch Zersetzung von Petroleumrückständen u. dgl. ertheilt, was ihm stets zugleich einen durchdringenden Geruch mittheilt.

Es scheint also in der That am Orte, wenn das hohe Industrie- und Landwirthschaftsdepartement zum Schutze der Arbeiter gegen Schädigung durch das Wassergas und Halbwassergas besondere Massregeln vorschreibt, immerhin unter der Voraussetzung, dass diese der Art seien, der Industrie keine hemmende Fessel anzulegen, und sie dadurch gegen die Nachbarländer zu benachtheiligen, wo überall die Anwendung des Wassergases und Halbwassergases ohne Beschränkung erfolgt.

Die Commission glaubt nun, dass solche Vorschriften sich in folgenden Richtungen bewegen können.

Erstens ist es höchst wünschenswerth, dass an der Hauptleitung des Gases ein Apparat angebracht werde, durch den man sich davon überzeugen kann, dass das Röhrensystem wirklich dicht ist und dass nach Abstellung des Haupthahnes nicht etwa Hähne offen geblieben sind, welche nach Wiederöffnung des Haupthahnes unverbranntes Gas ausströmen lassen würden. Man besitzt hierzu schon für den gleichen Zweck construirte Vorrichtungen, von denen der Muchall'sche Gas-Controleur (zu beziehen z. B. von Schmitz \& Morf in Zürich) im eidgenössischen Chemiegebäude überall eingeführt ist. Ein anderer einfacher Apparat für diesen Zweek ist von Dir. Rothenbach beschrieben. Beide Vorrichtungen sind sehr einfach, billig und leicht zu handhaben; sie erfüllen nicht nur den Zweck der hygienischen Controle, soudern sichern auch den Fabrikanten gegen Verluste. Ls ist nicht zu bezweifeln, dass die letzteren froh sein werden, auf diese Controle hingewiesen zu werden, die ihnen keine irgend nennenswerthe Ausgabe verursacht. Wir glauben, dass dies für die Consumenten von Wassergas und Halbwassergas obligatorisch gemacht werden sollte, und dass alsdann in Verbindung mit 
der zweiten Massregel Unfälle und Erkrankungen aus dieser Ursache so gut wie ganz ausgeschlossen sind. Übrigeus sollte der "Gascontroleur" so angebracht sein, dass auch ein Entweichen des Gases in den Boden (bei unterirdischen Leitungen) dadurch angezeigt wirl.

Zweitens empfehlen wir unbedingt, allen Fabrikanten vorzuschreiben, die Verbrennungsvorrichtungen für jene Gase so einzurichten, dass die Verbrennungsproducte aus dem Lokale abgeführt werden, wobei dann auch das recht häufig durch Zufälle mitkommende unverbrannte Gas mit beseitigt werden wird. Wenn es sich um Beleuchtungsvorrichtungen oder andere Verwenduvgen handelt, bei denen die Brenuer sich über Kopfhöhe befinden, so wird vermuthlich die schon aus allgemeinen hygienischen Rücksichten erforderliche Ventilation der Lokale für den genannten $\mathrm{Zweck}$ genügen, und wird es dann nur am Platze sein, diese Ventilation auch in Bezug auf die etwaige Anwesenheit von Kohlenoxyd zu controliren, z. B. vermittels Palladiumpapieres. Wo aber die Brenner bei oder unter Kopfhöhe angebracht sind, da wird es nöthig sein, dieselben mit Trichtern, Hauben oder anderen Vorrichtungen zu versehen, durch welche die Verbrennungsprodukte irgendwie aus dem Lokale heraus in's Freie geführt werden. Am sichersten wird das der Fall sein, wenn man diese Gase in einen Schornstein abführen kann, doch wird bei kleineren Mengen auch ein durch die oberste Fensterscheibe oder in gleicher Höhe damit geführtes Rohr genügen.

Drittens wäre es wünschenswerth, wenn auch nicht unbedingt nöthig und vielleicht auch zur Zeit noch nicht allgemein durchführbar, wenn man dem Wassergas und Halbwassergas auf künstlichem Wege einen penetranten Geruch ertheilen könnte, durch den ein Entweichen desselben sich von selbst verrathen würde. Für das eigentliche Wassergas sind in dieser Richtung von interessirter Seite schon sehr viele Versuche gemacht worden, und hat der erste Kenner dieser Industrie, Ingenieur Blass in Essen, dem unterzeichneten Prof. Lunge mitgetheilt, dass es nunmehr gelungen sei, das Wassergas vermittels eines höchst einfachen Apparates schon am Erzeugungsorte mit einer minimalen, aber für das Geruchsorgan unbedingt hinreichenden Menge von Mercaptan dauernd zu imprägniren. $\mathrm{Ob}$ sich dies in der That auch auf die Länge so verhält, werden die bei Gebr. Sulzer im Gange befindlichen Versuche wohl schon jetzt entschieden haben. Für das Halbwassergas ist eine ähnliche Imprägnirung mit Geruchsstoffen unseres Wissens bis jetzt noch nicht angewendet worden; wenn aber das Blass'sche Verfahren sich bewährt, so steht bei seiner Einfachheit und Billigkeit dessen Anwendung auch in diesem Falle sicher nichts entgegen. Eine etwaige Befürchtung der Erhöhung der Giftigkeit der Gase durch die Beimischung von Mercaptan ist ganz ausgeschlossen, da die Gewichtsmenge des letzteren so zu sagen unendlich klein, und durch chemische Reactionen gar nicht nachweisbar ist.

Auf den im Obigen mitgetheilten Thatsachen, Versuchen und Gründen fussend, beehren wir uns mithin, dem hohen Departement vorzuschlagen, folgende Bestimmungen für die dem Fabrikgesetz unterstellten Etablissements $\mathrm{zu}$ erlassen.

1) Die Anbringung von "Gas-Controleuren " oder ähnlichen Apparaten zur Controle des Entweichens von Gas aus undichten Stellen der Leitung oder offen gelassenen Hähnen wird allgemein verbindlich gemacht, wo Wassergas, Dowson-Gas oder ähnliche kohlenoxydreiche Gase in Leitungen verwendet werden. Die Controlapparate sind so anzubringen, dass sie schon Undichtheiten der Hauptleitung, namentlich auch deren unterirdischer Theile, anzeigen.

2) Es muss allgemein dafür gesorgt werden, dass die Verbrennungsproducte jener Gase, welche sehr leicht aus Zufall noch unverbranntes Kohlenoxyd enthalten können, sich der zum Athmen bestimmten Luft der Fabriklokale nicht beimengen können. In welcher Weise dies geschehen soll, ist nicht allgemein, sondern nur nach den speciellen Umständen jedes Einzelfalles zu bestimmen.

3) Es wird den Fabrikanten empfohlen, obige Gase durch Imprägnirung mit einem Riechstoffe weniger gefährlich zu machen. Von obligatorischer Einführung dieser Massregel wird zur Zeit noch abgesehen, bis längere Erfahrungen, namentlich von Seiten der Herren Gebrïder Sulzer vorliegen."

2. Juni 1888.

Bericht ïber die im Züricher HygieneInstitut ausgeführten Versuche über die toxische Wirkung des Wassergases und Halbwassergases.

Von

Prof. Dr. O. Wyss.

„Die zu diesem Zwecke angewendete Methode bestand darin, dass für Versuche von kurzer Dauer, d. h. von höchstens 11/2 Stunden, die Thiere in eine ihrer Grösse 